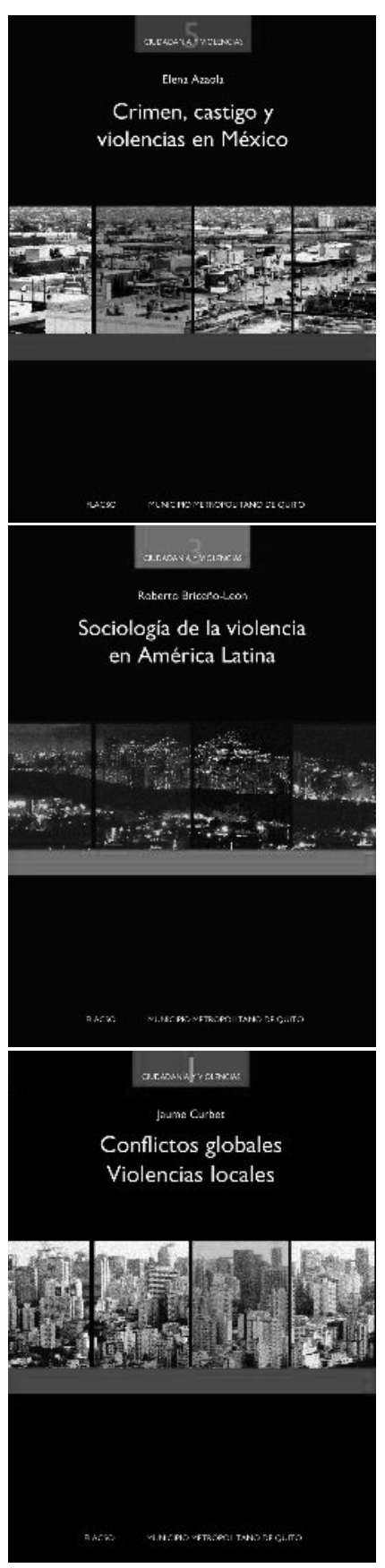

Fernando Carrión (Ed.)

\section{Colección Ciudadanía y} Violencias

Quito: FLACSO Sede Ecuador (2007-2008)
El crecimiento de la violencia y la inseguridad ciudadana es un fenómeno social de gran trascendencia que está afectando la vida de las personas a nivel mundial. No obstante, los niveles en que se expresa este fenómeno no son homogéneos. Ello ha generado un extenso debate que busca dictaminar las causas y posibles consecuencias de las múltiples violencias que aquejan nuestras sociedades. De igual manera se ha considerado necesaria la construcción de redes sociales e institucionales que aporten y refresquen conocimientos desde distintos lugares, perspectivas y enfoques para un mejor entendimiento de la naturaleza del fenómeno.

Es en este marco que la Facultad Latinoamericana de Ciencias Sociales (FLACSO), Sede Ecuador y el Municipio del Distrito Metropolitano de Quito, a través de la Corporación Metropolitana de Seguridad Ciudadana, plantearon el desarrollo de la colección "Ciudadanía y Violencias" cuyo objetivo es constituirse en una base bibliográfica que contribuya al conocimiento y debate sobre temas relacionados a la seguridad ciudadana a nivel mundial, pero con un claro énfasis en América Latina. Los 12 tomos de esta colección compilan los trabajos de autores y autoras internacionales de reconocida trayectoria en el análisis y reflexión de la violencia como fenómeno social, y de la seguridad ciudadana como propuesta de política pública que busca construir ciudadanía y mitigar los impactos de la violencia social. Así, esta colección atiende al desafío de generar herramientas de consulta académica e investigativa que puedan enriquecer, complejizar y democratizar el debate actual de la seguridad ciudadana. Hasta el momento se han impreso 6 volúmenes de la colección ${ }^{1}$.

El primero, Conflictos globales. Violencias locales, escrito por Jaume Curbet, director del Observatorio del Riesgo de Cataluña, nos permite tener una visión global sobre los problemas de inseguridad y su "localización" en contextos micro, específicos. Curbet inicia este volumen con una reflexión acertada: "persistimos en buscar la solución lejos de donde se halla el problema... buscamos la

\footnotetext{
Las reseñas de los volúmenes se apoyan también en los prólogos escritos por el equipo del Programa de Estudios de la Ciudad de FLACSO: Alfredo Santillán (Vol. 1); Daniel Pontón (Vol. 2); Andreina Torres A. (Vol. 3); Fernando Carrión (Vol. 4); Jenny Pontón (Vol. 5); Andrea Betancourt (Vol. 6).
} 

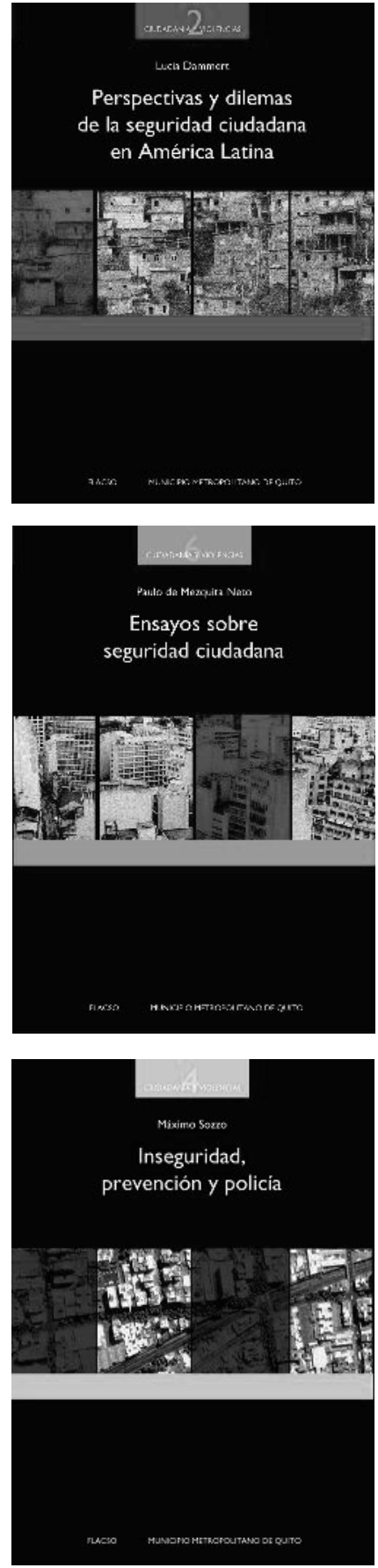

seguridad al margen de los problemas históricos y políticos, a la vez que psicológicos, que generan inseguridad" y esto sólo nos lleva a remedios técnicos y jurídicos que nos hacen evitar la discusión de fondo necesaria (Curbet 2007, p. 17). Para evitar caer en esta trampa, Curbet sugiere una revisión crítica de los riesgos inherentes al modelo de crecimiento económico predominante: el capitalismo occidental globalizado. Y de su corolario, la supremacía del "Yo separado" y la ambivalencia ante el deseo y el miedo. Bajo esta luz, el autor examina las dinámicas del Crimen Global Organizado, destacando su profitabilidad y escaso escrutinio en el mundo actual globalizado; la lógica de una "guerra contra el terrorismo" cuya inmediatez excluye cualquier tipo de reflexión acerca de sus tácticas; los riesgos producidos por la industria manufacturera, expresados, por ejemplo, en la abrumadora cantidad de accidentes de tránsito que desde esta óptica ya no resultan "accidentales"; y la dimensión social de los riesgos "naturales" cuyos impactos diferenciados revelan claramente la vulnerabilidad de los más pobres. Paralelamente, el autor analiza cómo estos procesos se "glocalizan", es decir, se traducen en problemas concretos en las localidades, generando fenómenos como los del "chivo expiatorio", que no es más que la materialización del miedo de los "ganadores" de la globalización frente a los "perdedores" o excluidos, muy claramente expresado en las reacciones ante la migración y la xenofobia que culpabiliza al de "afuera" por los problemas de inseguridad locales. Es por ello que una preocupación central del autor consiste en discutir la posibilidad de pensar una seguridad "sostenible", que ofrezca también la oportunidad de enfrentar los problemas de inseguridad no de manera sectorial sino de manera integral, a través de una mirada "penetrante, comprensiva y rigurosa". A pesar de la urgencia de las demandas por mayor seguridad, Curbet hace un llamado a la reflexión detenida y a poner un alto a la acción que termina por obviar la "raíz del problema".

El segundo volumen, Perspectivas y dilemas de la seguridad ciudadana en América Latina, presenta los trabajos de Lucía Dammert, quien dirige el Programa Seguridad y Ciudadanía de la Facultad Latinoamericana de Ciencias Sociales de Chile, y es considerada como una de las autoras de mayor impacto e influencia en el debate actual de la seguridad ciudadana en América Latina. Los estudios de la autora nos ofrecen un recorrido por una gran variedad de problemas que son organizados en dos áreas temáticas. La primera, titulada "Desafíos de la seguridad ciudadana en América Latina”, explora problemáticas 
que se expresan con ciertas similitudes en la región y han sido objeto de arduo debate en los últimos años. Tales son, los desafíos inherentes a los esfuerzos de prevención del delito a través de la participación comunitaria; la reforma policial y la militarización de la seguridad pública; y el rol de los medios de comunicación en el tratamiento de la violencia. También introduce en esta sección un tema relativamente inexplorado: la diferenciación por género de las percepciones de inseguridad, analizando el temor entre las mujeres.

En una segunda sección del volumen, Dammert se concentra en la problemática de Chile y Argentina. Aquí la autora explora la realidad de la violencia criminal y la victimización en Chile, a través de un análisis de las fuentes estadísticas disponibles; las reformas que ha sufrido la seguridad pública en Chile entre 1973 y 2003; los desafíos del sistema carcelario y los riegos implícitos en la respuesta de privatización de las cárceles; la relación entre jóvenes, violencia y policía en Chile; y finalmente, los efectos del temor en la configuración territorial de las ciudades y en los niveles de confianza en la policía.

No cabe duda que, en este volumen de la colección, Dammert aborda algunos de los temas centrales de discusión en la comprensión de la violencia y las reformas necesarias para su adecuado manejo institucional o a través de nuevas configuraciones que se están generando con la participación de la ciudadanía, siempre tomando en cuenta los límites y desafíos implícitos en este tipo de esfuerzos.

En el tercer volumen, Sociología de la violencia en América Latina, compila los trabajos que Roberto Briceño-León ha desarrollado durante su larga y fructífera trayectoria científica y en su calidad de director del Laboratorio de Ciencias Sociales (LACSO) con sede en Caracas, Venezuela. Los análisis del autor se concentran en un campo importantísimo de la agenda de investigación de la violencia y la inseguridad ciudadana: la comprensión de los fenómenos violentos desde una perspectiva sociológica. Dentro de este objetivo general podemos desglosar algunos campos importantes de reflexión. En primer lugar, es claro que Briceño-León demuestra una fuerte preocupación por desarrollar marcos explicativos para entender la violencia en América Latina, como un fenómeno esencialmente urbano. Es así que el autor propone su ambicioso "marco sociológico para explicar la violencia urbana”, una propuesta que, como reconoce el mismo autor, no deja de tener limitaciones pero puede ofrecer ciertas luces para comprender un fenómeno que comprende múltiples aristas. En esta línea, para el autor resulta esencial identificar ciertos fenómenos y estudiarlos desde una perspectiva sociológica, como la "violencia juvenil" entendida como el producto de la exacerbación de expectativas de consumo y calidad de vida en contextos que ofrecen escasas oportunidades a este grupo etáreo. Otra arista que sobresale en los análisis de Briceño-León, se refiere a los efectos devastadores de la violencia en la convivencia y el Estado de Derecho, los cuales se ven reflejados en actitudes que apoyan el "derecho a matar", el fracaso del proyecto "civilizatorio" y, por último, el miedo y la "pérdida" de la ciudad. Finalmente, las reflexiones del autor se concentran en desentrańar la especificidad de la violencia en Venezuela, en el marco de los procesos políticos que ha experimentado el país en los últimos años.

De esta manera, Briceño-León nos ofrece un volumen que aporta una entrada interesante para entender las dinámicas de la violencia urbana en la región y, más aún, para comprender 
los devastadores efectos que la misma está teniendo en la construcción de ciudadanías más tolerantes, pacíficas y respetuosas del Estado de Derecho.

Inseguridad, prevención y policía, escrito por Máximo Sozzo, constituye el cuarto volumen de la colección. Sozzo, quien es profesor de la Universidad Nacional del Litoral de Argentina, nos presenta indagaciones teóricas enmarcadas en el campo de la criminología y la sociología. Su mirada analítica nos lleva por recorridos teóricos, históricos y geográficos, así como por estudios empíricos novedosos. Uno de los temas analizados por el autor se concentra en las dificultades que impone la medición del delito o, en palabras del autor, la "tarea de conocer" el fenómeno de la criminalidad. En este campo Sozzo hace un abordaje crítico de las diversas herramientas existentes para un acercamiento "objetivo" a las cifras del delito, con un especial énfasis en las encuestas de victimización y su aplicación en diferentes contextos. Otra tarea que emprende el autor consiste en develar los preceptos teóricos e ideológicos y las racionalidades que se esconden tras las diversas propuestas de prevención del delito, llamando la atención sobre el origen de las mismas y su lugar de enunciación, para de esta manera poder mantener un ojo crítico ante respuestas que pueden presentarse como meramente técnicas y libres de valores. Apoyado en las ideas de Crawford, Sozzo hace una disección de las "tácticas" de prevención del delito - "situacional y ambiental", social y comunitaria- y sus diversas "técnicas de intervención", tomando ejemplos de diferentes países, principalmente anglosajones. El autor también se muestra interesado en estudiar los "viajes culturales" de las tácticas y técnicas de prevención del delito, al hacer un estudio pormenorizado de la importación/traducción cultural del modelo de Neighbourhood watch en un barrio de la Ciudad de Buenos Aires, que nace como una iniciativa de individuos preocupados por el delito en su comunidad y luego se institucionaliza a través del Plan Nacional de Prevención del Delito en Argentina. Por último, un importante eje de análisis que nos presenta el volumen de Sozzo se relaciona con el rol histórico que ha tenido la policía, las diversas lógicas y racionalidades que han definido su accionar y los procesos de reforma policial en el marco de las estrategias de prevención del delito. En este sentido, el volumen abre nuevas vetas de análisis y nos presenta un enfoque particular enmarcado en la criminología crítica.

Por su parte, Elena Azaola, la autora del quinto volumen Crimen, castigo y violencias en México, nos muestra el trabajo que ha venido realizando a lo largo de los últimos ańos como antropóloga y psicoanalista interesada en diversas problemáticas que tienen como eje común la comprensión de la violencia y la criminalidad y las distintas instituciones que rodean al fenómeno, en el contexto particular de México. Gran parte de los estudios de Azaola se destacan además por incorporar una perspectiva de género e incorporar como sujetos de estudio a grupos que frecuentemente se hallan sub-representados en los debates sobre la seguridad ciudadana, tales como las mujeres y los/as niños/as. Las preocupaciones de Azaola también se encuentran fuertemente enmarcadas en el ámbito de los derechos humanos y la necesidad de recuperarlos como marco básico para cualquier esfuerzo de reforma o cambio en el ámbito de la seguridad pública.

A más de este marco general en el que se puede ubicar el trabajo de la autora, el volumen desarrolla varios temas específicos que vale la pena destacar. Uno de ellos es el funcionamiento de la policía, como una de las principales instituciones encargadas de garantizar la seguridad pública, específicamente en la ciudad de México. En esta área Azaola señala las ya conocidas deficiencias estructurales de corrupción y falta de equipamiento de los policíal, pero quizás los más importante 
e interesante del trabajo de la autora es su acercamiento etnográfico a las distintas percepciones que los oficiales tienen sobre la institución, sobre sí mismos como policías y sobre sus superiores. Otro tema que se analiza es el funcionamiento del sistema penitenciario en México, y en este contexto introduce problemáticas específicas como la criminalización femenina y la diferenciación por género en el tratamiento que la justicia hace de delitos como el homicidio. Otro interés que vemos reflejado en el volumen, es el análisis de distintas formas de violencia, tales como la violencia de género y el maltrato infantil, temáticas que analiza también desde una óptica trasnacional al incorporar, por ejemplo, el tráfico y la explotación infantil en zonas transfronterizas. De esta manera, Elena Azaola nos abre un abanico de posibilidades que amplía la discusión y plantea nuevos retos a la agenda pública en materia de seguridad ciudadana.

El último volumen publicado hasta ahora, titulado Ensayos sobre seguridad ciudadana, constituye para FLACSO Ecuador un tributo al legado de Paulo Mesquita Neto, reconocido académico del Núcleo de Estudos da Violência de la Universidad de Sao Paulo, Brasil, quien lamentablemente falleció el presente año. Los trabajos de Mesquita Neto giran en torno a varios ejes prominentes. Uno de ellos es el contexto de la democratización en Brasil y lo que ésta ha significado en términos de cambios y continuidades en el sistema de seguridad pública del país. Mesquita Neto es claro en enfatizar las resistencias que han enfrentado los distintos esfuerzos de reforma que se han tratado de impulsar en el país y la persistencia (antes que transformación) de una lógica militar que ha dominado el sistema de seguridad pública brasileño. De cara a los crecientes niveles de inseguridad, que también son documentados por el autor, la situación es preocupante.

Es así que un segundo nodo de análisis, se ubica en el campo de las políticas públicas y, principalmente, en la compresión del rol que los municipios empiezan a jugar en el ámbito de la seguridad pública o ciudadana en el país, esfuerzo que para el autor se materializa en la creación de instancias de discusión y acción como el Foro Metropolitano de Seguridad Pública de Sao Paulo. Un tercer nodo de análisis importante, es la reafirmación de los derechos humanos como un marco básico necesario para exigir una revisión de las prácticas policiales y una reducción efectiva de la violencia policial. Mesquita Neto hace una revisión minuciosa de los presupuestos asignados al Plan Nacional de Derechos Humanos de Brasil y comprueba la todavía insuficiente importancia que se ha dado al tema, incluso bajo la administración Lula. Por último, una temática que marca gran parte de los estudios desarrollados por el autor se refiere al rol de la policía, su funcionamiento en el contexto particular de un país federal, las investigaciones que se han producido sobre la institución en las últimas décadas y las posibilidades de reforma de dicha instancia en Brasil en el período democrático. Así, por ejemplo, el autor estudia con detenimiento las experiencias de policía comunitaria en la ciudad de Sao Paulo, para señalar las bondades pero también los múltiples desafíos que plantea la implantación de un modelo de este tipo en una de las ciudades más importantes de Brasil.

Para Mesquita Neto, ninguna de estas preocupaciones se presenta de manera aislada, constituyen partes de un todo coherente que finalmente debería expresarse en una reforma profunda del sistema de seguridad pública no sólo en Brasil sino en toda América Latina. El legado de Mesquita Neto es invaluable y constituye una referencia obligada para cualquiera que desee acercarse a la realidad brasileña en esta materia pero también para aquellos que deseen explorar nuevas posibilidades de conceptualización y comprensión de la seguridad ciudadana. 
Como es posible apreciar, la colección Ciudadanía y Violencias recoge una multiplicidad de enfoques que reflejan, a su vez, la diversidad geográfica de sus contribuyentes. A pesar de ello, es posible también identificar ciertas líneas de investigación comunes, que se han convertido en temas importantes de debate en la región en los últimos años: los crecientes niveles de inseguridad y violencia en la región; la prevención del delito y la participación de la comunidad y de los gobiernos locales en este campo con sus múltiples ventajas pero también desafíos; la reforma de la policía y de otras instituciones que tradicionalmente han sido responsables por la seguridad pública o "ciudadana"; la transición democrática como marco histórico y político para entender las nuevas tendencias y cambios en el debate sobre la seguridad y su conceptualización como un bien público común; las dificultades presentes en los esfuerzos por "medir" la violencia y la inseguridad; y los efectos de la "inseguridad", en tanto percepción y no realidad objetiva, entre otras. No obstante, el abordaje de cada uno de estos temas en los distintos volúmenes refleja una gran heterogeneidad y múltiples posibilidades de análisis de un mismo problema.

Paralelamente, los diversos textos recogidos en esta colección nos permiten acercarnos a temas relativamente inexplorados tanto a nivel regional como a nivel global, como lo son: las implicaciones locales de los conflictos globales; el estudio de las violencias que no son comúnmente asociadas a la inseguridad delictual; la incorporación de una perspectiva de género al análisis de las violencias y los miedos urbanos; la exploración etnográfica de instituciones como la policía; los "viajes culturales" de las ideas que se van adoptando como políticas y acciones de seguridad ciudadana, entre otros. Los diversos volúmenes invitan a expandir la agenda de investigación y de debate hasta ahora establecido y a continuar abriendo nuevas vetas de análisis y reconociendo los vacíos aún presentes, no sólo en la comprensión sino también en la acción en torno a este ámbito.

Es por esto que la colección "Ciudadanía y Violencias" constituye un aporte esencial para el debate actual sobre la violencia y la inseguridad, e invitamos a los y las lectoras a estar atentos a los próximos volúmenes que serán publicados, y que incluyen a académicos del calibre de Hugo Frühling (Chile), Carlos Mario Perea (Colombia), Michel Wieviorka (Francia), Máximo Pavarini (Italia) y Fernando Carrión (Ecuador).

Andreina Torres Angarita*

\footnotetext{
* Profesora-investigadora asociada al Programa de Estudios de la Ciudad de FLACSO Sede Ecuador. Coordinadora editorial de la Colección Ciudadanía y Violencias.

Correspondencia: Andreina Torres Angarita, FLACSO, La Pradera E7-174 y Diego de Almagro, Quito, Ecuador. E-mail: antorres@flacso.org.ec.
} 